\title{
Improving simulation debriefing in paramedic education: the Paramedic Debrief Model
}

Alan Wilson DipTchg, DipNsng, GradDipIntensiveCareParamedicine, PGDipClinEd, MProfPrac(Ed), MPA is Paramedic Lecturer'; Elizabeth Ann Asbury BSc(Hons), MSc, PhD, MPA is Academic Project Leader ${ }^{1}$

Affiliation:

${ }^{1}$ Whitireia New Zealand, Auckland, New Zealand

https://doi.org/10.33151/ajp.16.656

\section{Abstract}

\section{Introduction}

Simulation training is widely used in paramedic education. Quality of debriefing is central to optimising learning from educatorfacilitated simulation training. There is considerable variation in the provision of simulation debriefs by paramedic educators, often leading to a varied perception of the quality of debrief by both students and tutors. There are currently no standardised debrief tools specific to paramedic practice. The purpose of this study was to provide a rationale and theoretical framework for the development of a paramedic specific model for simulation debrief, the Paramedic Debrief Model (PDM), that reflects the key elements of optimal debrief practice, feedback and reflective learning principles and that is aligned to a construct of paramedic competencies.

\section{Methods}

A systematic literature review was conducted that focussed on two areas: best practice, principles and models for simulation debrief and constructs for paramedic competencies. Emergent theme analysis was used to identify key components of effective simulation debrief and a construct of paramedic competencies that could be incorporated into the debriefing tool.

\section{Results}

It was identified that an optimal simulation debrief transitions sequentially through the elements of reaction, recollection, reflection, analysis and application. The construct of paramedic competencies best aligned with paramedic education consist of eight elements: communication, resource utilisation, ethics, situational awareness, decision making, patient assessment, history gathering and procedural skills. These themes were combined to create the PDM.

\section{Conclusion}

The study identified the key elements of an optimal simulation debrief and incorporated a construct of paramedic competencies to create the PDM. The PDM offers an evidence-based, structured approach to paramedic simulation debriefing and has potential to be utilised as an optimal strategy for facilitating simulation debriefs in paramedic education.

Keywords:

constructive alignment; debrief; education; paramedic; simulation

Corresponding Author: Elizabeth Asbury, Elizabeth.Asbury@whitireia.ac.nz 


\section{Introduction}

High fidelity simulation training - defined as the use of high fidelity computerised manikins to simulate patient responses in real life scenarios - has demonstrated expediential growth in the past decade as a training method to improve clinical skills, knowledge and patient safety across a wide spectrum of healthcare disciplines and specialties (1). Simulation training is seen to have many benefits. It allows for standardisation and consistent replication of patient conditions; learners are able to make errors, learn from mistakes in a risk free environment, and cause no harm to real patients (2). Simulation negates the need to gain consent from patients (3), enables training that focusses specifically on development of strategies to avoid medical error (4), and permits students exposure to conditions that would be rarely encountered in the clinical environment (5). Simulation also offers an alternative solution for clinical exposure with the increasingly limited opportunity for students to gain clinical placements in hospitals or clinical environments (2).

\section{Simulation training is grounded in constructivism learning} theory (6), which asserts that through an experiential learning process centred on reflective learning practices $(7,8)$ learners will learn, develop, construct and reconstruct learning. Simulation training as a pedagogy provides a framework for this learning to take place. Therefore, there is a strong pedagogical as well as ethical imperative for simulation training to be a regular component of healthcare education programs $(3,5)$.

Simulation training is a widely utilised training methodology in paramedic education $(9,10)$. Paramedic education institutions report high levels of simulation training in their programs, utilising a wide range of simulation manikin technology from simple task trainer manikins through to advanced and fully programmable simulation manikins (2). Paramedics and paramedic students report high levels of satisfaction with simulation training $(11,12)$, however, both paramedic and healthcare educators identify a lack of simulation-specific training as being the biggest barrier to its effective utilisation $(1,2)$.

Central to optimising learning from the simulated training environment is the quality debriefing, which is widely acknowledged as being the most important component of simulation training (1). Quality debriefing promotes good reflective thinking on behalf of the learner and is further enhanced by good feedback from the facilitator.

Educators have a crucial role to play in facilitating this reflective learning process in the simulation debrief where construction and reconstruction of learning takes place. Evidence suggests that a structured approach to debrief is better than an unstructured approach $(13,14)$. A structured debrief format has been more positively perceived by learners and also yields more positive results with measured learning outputs when compared to unstructured 'ad hoc' style debriefs $(14,15)$.

It has been observed that there is considerable variance in the way debriefs are conducted by paramedic educators, often leading to a varied perception of the quality of debrief by both students and tutors. There is a paucity of research on the optimal structure, model and process for simulation debriefs, particularly in relation to paramedic education and simulation debrief. However, there has been a small but growing body of academic literature from the medicine, nursing and in-hospital training context suggesting that the consistent utilisation of a structured debrief model improves the quality of simulation debriefs and educational outcomes (14). Moreover, research suggests that an optimal debrief process needs to be aligned to the actual simulation experience and the predetermined purpose of the simulation (16). This concept is supported by constructive alignment curriculum theory (17). This theory asserts that learners engaged in any learning activity should have a clear understanding of the intended learning outcomes and the teaching or learning activity and any related assessment should be aligned to these outcomes (17). In the simulation learning context, the progression of a learner should start by understanding the intended learning outcomes, participate in the simulation experience, and then through a facilitated debrief and formative feedback process aimed at reflection and analysis, reconstruct learning. The intended learning outcomes are aligned with the learning activity (simulation experience), which informs the formative feedback (debrief process) (18). Ideally these intended outcomes should reflect a balanced approach between addressing the individual learning needs of the students and the intended learning outcomes of the paramedic education curriculum (19). Ideally, intended learning outcomes should also be aligned ultimately to paramedic attributes, performance domains and competencies (17).

The purpose of this study was to provide a rationale and theoretical framework for the development of a paramedic specific model for simulation debrief, the Paramedic Debrief Model (PDM), that reflects the key elements of optimal debrief practice, feedback and reflective learning principles and that is aligned to a construct of paramedic competencies. The aim of this article is to outline the rationale and theoretical framework for the development of the PDM that was drawn from a systematic review of literature related to simulation debriefing and paramedic competencies (Figure 1). Ultimately the use of the PDM will allow for a consistent and structured approach to the debrief after a simulated clinical experience that is specific to the needs and practices of paramedics. This will lead to better outcomes for paramedics and paramedic students in terms of fostering optimal reflective practice skills that will improve paramedic competence and confidence. This could also, in turn, lead to better outcomes for patients. 
The intention of the PDM is to provide a tool that paramedic educators can use as an aide memoire, that captures the key principles of optimal simulation debrief, when facilitating paramedic simulation debriefs with undergraduate paramedics students. However, the model's generic approach to capturing core principles of optimal debrief as well as core generic paramedic competencies enables the PDM to be applied in the facilitation of simulation debriefs among established and practising paramedics. The PDM encapsulates the core principles that should be addressed in any simulation debrief, regardless of the mode of delivery. Facilitation of simulation debrief involves a broad range of delivery modes and technology ranging from the use of video technology through to live multi-centre streaming through to various ways of fostering self-reflection or peer review. The core principles of the PDM would apply regardless of the mode of debrief facilitation.

\section{Methods}

In order to explore simulation debriefing in the context of paramedic education, and a construct of paramedic competencies, a literature review was completed in March 2017 using the following data bases: PubMed, ProQuest, Science Direct and Google Scholar. The review sought literature in two areas related to the main research objective: best practice, principles and models for simulation debrief; and constructs for paramedic competencies. Search terms included various combinations of 'paramedic', 'ambulance', 'pre-hospital', 'simulation', 'simulation training', 'high-fidelity simulation', 'education', 'debriefing', 'debriefing models', 'research' and 'paramedic competencies'. Initial inclusion criteria included a) research that focussed on paramedic simulation debrief, b) research that focusses on paramedic competencies, c) primary and secondary research studies, d) peer reviewed commentary

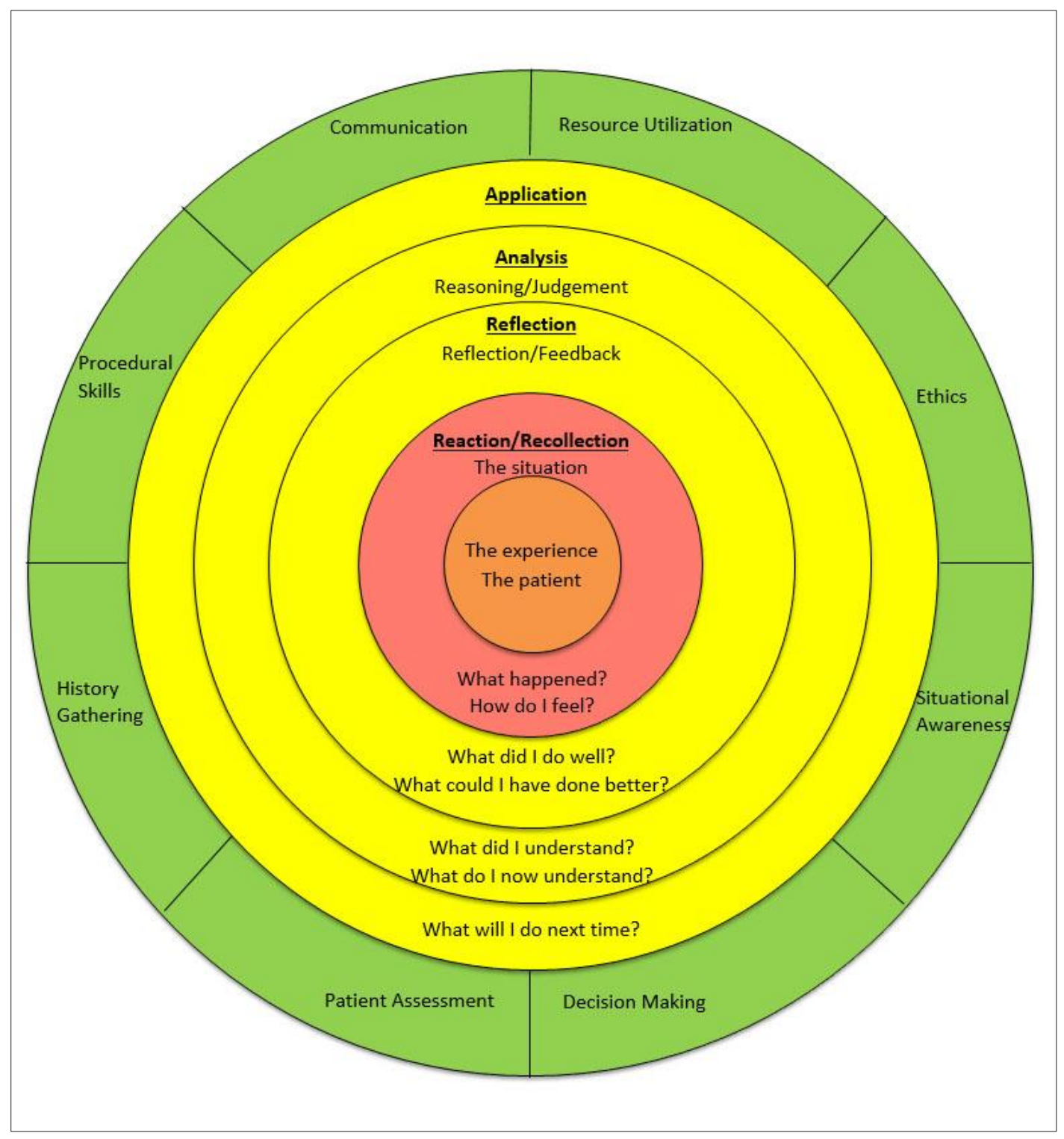

Figure 1. Paramedic Debrief Model 
articles, e) studies published later than 2000, and f) studies in English language only. An initial search revealed a lack of literature directly related to simulation debrief and paramedic education. As a result the inclusion criteria was broadened to research on healthcare simulation debrief. Selected studies were further grouped for suitability under the following headings related to the research question and areas of interest: a) paramedic simulation training, b) principles and practices of simulation debrief and debrief models, and c) construct of paramedic competencies. Seven articles were found relating to paramedic simulation training, 19 articles relating to principles and practices of simulation debrief and debrief models, and seven documents relating to paramedic competencies.

Emergent theme analysis of the literature was undertaken to identify key components of an effective debriefing process and identify a construct of paramedic competencies. This in combination with an understanding of existing constructive and reflective learning theories (6-8) and constructive alignment curriculum theory $(17,20)$ informed the development of the PDM.

\section{Results}

Thematic analysis of the literature was broadly divided into those themes related to simulation debrief practices and principles and then those related to paramedic competencies. These themes were assimilated into the development of the PDM.

\section{Debrief practices and principles themes}

A small number of studies had developed models or structures for simulation debrief (15,21-27). Four themes were identified through an emergent thematic analysis of the models that reflect the structural elements and sequential phases of an optimal debrief process. These themes were:

\section{Phase 1: Reaction/recollection \\ 2. Phase 2: Reflection \\ 3. Phase 3: Analysis \\ 4. Phase 4: Application.}

These themes were consistent with constructive learning theory and experiential and reflective learning concepts. These elements informed the development of the structural elements and sequential phases of the PDM (Figure 1).

\section{Phase 1: Reaction and recollection}

All the literature referred to a debrief process beginning with participants being encouraged to react to the simulation and then recall what happened. The focus is on participants considering and identifying the impact of the experience and recalling or describing what actually happened. This phase is referred to in the literature as the descriptive $(22,28,29)$, defusing $(24)$, or reaction $(23,25,27,30)$ phase. The purpose of this phase is for learners to express their initial emotional reactions to the event as well as observational recollections of the actual experience, what happened and in what order.

The reaction phase enables feelings, emotions and immediate reactions related to the experience to be expressed, allowing learners to move on, think clearly about the experience, or begin to reflect and analyse. 'Venting of feelings and emotions becomes an important defusing process. This process often begins naturally as participants leave the simulation and begin talking about the experience (24). The facilitators role is to continue this natural 'venting' process during this reaction phase of the debrief, identifying concerns that can be addressed in the subsequent discussion $(23,24)$.

The second aspect of this phase is recollection of what happened during the simulation experience. The goal here is for participants to describe what happened but with no attempt to analyse or reflect. The use of open-ended narrative questions will invite participants to comment on and recollect the event and facilitate a shared understanding of the experience (23).

\section{Phase 2: Reflection}

The second phase of the debrief process identified in the literature broadly relates to the theme of reflection. The focus is on the participants reflecting on their performance in the experience. This phase is referred to in the literature as the explain (15), reflection (30), analysis and analogy $(22,28,29)$ or discovering (24) phase. The purpose of this phase is for participants to focus and reflect on their own performance and also the performance of the team. This phase aligns with Schons' 'reflection on action' phase of reflective learning (7). It also aligns with both the reflective observation stage of the experiential learning cycle (6) and evaluation in the reflective learning cycle (8).

Primarily, the focus is on the participant becoming self-aware and identifying the positive and negative aspects of their performance, while receiving debrief feedback. The Pendleton Clinical Feedback Model that focusses on what went well and what could have been improved is a commonly utilised tool that forms a useful structure for this reflective phase and assimilates both a participants self-reflection with feedback from the debrief facilitator (31). It has been argued that when using this model, reflection should start with the positive, move onto the areas for improvement and then finish with a positive (31).

\section{Phase 3: Analysis}

The third phase of the debrief process identified in the literature broadly relates to the theme of analysis. This focusses on the participants analysing their performance and more specifically their understanding and mental models that influenced their actions in the experience. Through this analysis participants may modify their understanding or identify new learning and understanding and is referred to in the literature as the 
analysis $(22,25,27,30)$, elaborate/evaluate (15), analogy/ analysis $(28,29)$ or discovering $(24)$ phase. This aligns with the reflection in action phase of the reflective learning model (7) and the analysis phase of the reflective learning cycle (8). This phase also equates with the abstract conceptualisation phase of the experiential learning cycle (6). A key focus for the debrief facilitator during this phase is to assist the learners in recognising the mental models, frames and decision making processes that lead to the actions identified in the reflection phase. A number of facilitation strategies and techniques are suggested for assisting learners to identify and process these mental models, including 'debriefing with good judgement' or advocacy inquiry (32), guided self-correction $(23,33)$, the systemic constructivist approach (23), directive feedback and teaching (25) and the analogical reasoning approach (24).

\section{Phase 4: Application}

The fourth and final phase of the debrief process identified in the literature broadly relates to the theme of application. It focusses on the participant considering how the experience and learning could apply to future clinical situations. This phase is referred to in the literature as the application $(22,25,27-30)$, extend (15), planned action (34) or deepening (24) phase. This aligns with the reflection beyond action phase of the reflective learning model (7) and the planned action phase of the reflective learning cycle (8). This phase is principally focussed on the application of new and reworked understandings that have been identified in the reflection and analysis phases.

It is important in this phase to draw explicit connection between the reconstructed learning and practice in the actual clinical environment. This phase would equate to the active experimentation phase of Kolb's Experiential Learning Model (6). Kolb would argue that it is important in this phase for the learner to explore the new learning by immediately experimenting with it through, in the case of simulation, a repeat simulation experience.

Having the patient at the centre of the PDM is a reminder to paramedic simulation students that the patient and patient focussed principles should always be central in their care and actions $(35,36)$. In addition, a principle central to constructivism learning theory is that constructive learning takes place through actively engaging in an experience (6). The core focus and origin for experiential learning theory is the concrete experience. The very centre of the PDM, therefore, is the experience/patient. This reinforces the concept that the experience associated with the patient encounter is the primary focus and the primary catalyst for the subsequent construction and reconstruction of learning facilitated through the debrief.

\section{Paramedic competency themes}

Although most paramedic services, organisations and professional bodies will have a list of paramedic competencies (37-41), the literature revealed surprisingly little research on the construct of paramedic competencies. Tavares and colleagues developed a valid construct of paramedic competencies in the context of developing a global rating scale for the assessment of paramedic clinical competence (42). Using a robust process involving task analysis, focus groups and feedback, input and analysis from leading local and national paramedic experts via a modified Delphi process, seven dimensions with accompanying descriptions were identified that represent the overarching construct of paramedic competencies. The resulting final construct of competency dimensions as:
1. Situation awareness
2. History gathering
3. Patient assessment
4. Decision-making
5. Communication
6. Resource utilisation
7. Procedural skills.

Tavares et al applied these constructs to a global rating scale (GRS) assessment matrix applying it to sample performances in a simulated scenario from three groups: novice paramedic students, entry-to-practice level paramedic students and experienced paramedics (42). The paramedic competency dimensions and the GRS have been successfully used as an assessment tool for evaluating paramedics in both a simulation based assessment and workplace based assessments in the real clinical environment (43). Therefore, the competencies and descriptors developed by Taveres et al were utilised in the PDM (Figure 2). It is interesting to note that the paramedic competencies developed by Taveres et al are very similar to the performance domains developed and utilised in the PEARLS Healthcare Debriefing Tool that has been developed as a debrief tool for general healthcare simulation (25-27).

An additional eighth dimension, 'Ethics', was added to the competencies in the PDM. Emerging professionalisation has enabled paramedicine to adapt core healthcare competencies from the earlier professionalisation of allied healthcare disciplines such as nursing and medicine $(44,45)$. One such competency is an understanding of healthcare ethical principles, regulatory frameworks and legislation that have jurisdiction over healthcare delivery. This is an essential competency for any healthcare professional, including paramedics (44). In particular, having the skills and knowledge to navigate the complexities of legal and ethical dilemmas that present in any healthcare situation is an important skill set for paramedics (44). In addition, an understanding of and ability to apply ethical and legal principles is a required professional competency standard under paramedic jurisdictions, professional bodies and standards $(40,41)$. It was therefore considered an important competency dimension to add to the PDM.

\section{Application of the Paramedic Debrief Model}

In terms of application and use of the PDM, the experience/ patient is at the centre of the model. The intention is that both the debrief facilitator and learners are aware that the principal focus of the debrief is the patient. The model then is designed 


\section{Situational awareness}

\section{Paramedic Compentencies ${ }^{1}$}

Refers to the individual's overall ability to consider and integrate environmental, scene, resources and patient condition cues into overall interaction, management and safety plan. This includes observing whole environment (all available sources), anticipating likely events, discriminating between relevant and irrelevant data and avoiding tunnel vision (inappropriately focusing on elements to the exclusion of others). The individual is expected to demonstrate examples of situation awareness throughout the interaction and updating actions as necessary.

\section{History gathering}

Refers to the individual's overall ability to effectively and thoroughly gather an appropriate history (includes history of present illness and medical history) which is organised appropriately structured, timed and focused according to the clinical situation and level of urgency (context). This includes interpreting and evaluating findings while discriminating between relevant and irrelevant findings. Also refers to a demonstrated ability to include a consideration for differential diagnosis while working towards a working diagnosis.

\section{Patient assessment}

Refers to the individual's overall ability to select and perform a physical exam and investigation of signs and/or symptoms that is organised and appropriate given the clinical situation and level of urgency. This includes interpreting and evaluating findings while discriminating between relevant and irrelevant findings. Also refers to a demonstrated ability to continue appropriate reassessment/ detailed assessment as needed. Finally this includes a consideration for differential diagnosis while working towards a working diagnosis.

\section{Decision making}

Refers to the individual's overall ability to select an appropriate, safe and effective management plan and/or strategy. Decisions should be based on and supported by findings, considerations of risks, benefits and differential diagnosis. This involves having adequate information for decisions made (ie. avoiding premature closure) and ensuring that decisions are appropriately prioritised and timed. This includes selection of an appropriate management device, method or technique based on evidence (ie. situational awareness, patient condition, resources etc.) and context.

\section{Resource utilisation}

Refers to the individual's overall ability to identify and use resources effectively to accomplish goals and maximise care. This includes the delegation of tasks, the coordination of efforts, selecting appropriate members (eg. allied agencies, patients etc.) for a given task, ensuring effectiveness and requesting additional resources as necessary. This also includes ability to function as a team with appropriate leadership.

\section{Communication}

Refers to the individual's overall ability to clearly and accurately exchange information with the team, patient and/or bystander for optimal patient care and team effectiveness. This includes the use of concise and appropriate language, ensuring statements are directed at appropriate individuals and that messages are heard/received (ie. close the loop). This also includes demonstrating effective listening skills, demonstrating empathy, responding appropriately to statements by the team, patient or bystander. Actions are appropriately communicated with the team, patient and bystander. Verbal and non-verbal are appropriate and congruent.

\section{Procedural skills}

Refers to the individual's overall ability to complete psychomotor skills or tasks effectively, appropriately and to standard. This involves a familiarity with equipment used, ensuring appropriate and safe application while completing tasks to standard and avoiding commission or omission errors. This includes adaptability to failures/problems (as necessary) and ensuring team, patient or bystander safety while performing these procedures: includes appropriate execution, properly sequenced and evaluating/ revaluating effectiveness.

\section{Ethics}

Refers to the individual's overall ability to deliver care in an ethically and legally appropriate manner. This includes that all care follows the principles of beneficence, non-maleficence, autonomy and justice. This also includes ensuring that all care is delivered according to the principles and rights as outlined in the New Zealand Health and Disability Services Code of Consumer Rights.

${ }^{1}$ Adapted from Tavares W, Boet S, Theriault R, Maliette T, Eva KW. Global rating scale for the assessment of paramedic clinical competence. Prehosp Emerg Care 2013;17:57-67.

Figure 2. Paramedic competencies 
to allow the debrief facilitator to sequentially work through the respective phases of an optimal debrief, commencing with reaction and recollection, then reflection followed by analysis, and then finally application. Each of these phases has some suggested open ended questions that are designed to shape discussion and focus on the purpose of the respective phase. The paramedic competencies or performance domains around the outside of the model are a reminder that each simulation experience and therefore debrief should be focussed on one or more of these domains in accordance with constructive alignment principles. These should be reflected in the aim, objective and purpose of the simulation experience.

\section{Discussion}

Simulation training and the use of its associated technology is now an established training pedagogy in paramedic education $(2,10)$, positively perceived by both paramedic students and tutors $(11,12,46)$. It brings with it a focus on experiential learning that is concentrated on promoting reflective education that leads to construction and reconstruction of learning. It is widely acknowledged that a good debrief is critical to fostering this learning process and is arguably the most important component of the simulation training $(1,28,47)$. To date, much of the focus in paramedic simulation training has been on scenario design, its use in skill acquisition, task training and as an assessment tool (10). There has been little focus on the importance and place of debrief. Skilful debrief that focusses on fostering reflective learning and construction and reconstruction of learning needs to follow a sequential process. Having educators skilled, equipped and trained in good debrief process is essential to this. The development of the PDM offers a unique and evidence based approach to debrief facilitation for paramedic educators to use.

The underpinning purpose behind the development of the PDM was two-fold. First, to provide a structured debrief model that has sequential phases that reflect best practices and principles for debriefing simulations. Second, that the model should follow constructive alignment principles by relating the intended learning outcomes to the simulation experience and a construct of paramedic competencies or performance domains. This constructive alignment approach is one of the new and unique features of this debrief model that also makes it unique to a paramedic practice context. The advantage of having a constructively aligned approach to simulation debrief is that student learning is specified and focussed, and that the debrief can be aligned to the specified intended learning outcomes. Using the paramedic competency dimensions from the work of Tavares et al (42) ensures the simulation experience is aligned to paramedic competencies and learning outcomes. This alignment is considered to help students in learning and also lead to better learner performance (17).

Constructive alignment theory holds that for optimal learning, learners need to be actively engaged in a learning/teaching activity or experience (17). Moreover, the learners need to have a clear understanding of the purpose or focus of the activity or experience through clearly defined learning outcomes. In the case of paramedics, these outcomes and competencies should be aligned to how paramedics practice in the day-today clinical environment. These in turn are determined by competency or performance domain frameworks formed by paramedic organisations or relevant professional regulatory bodies (Figure 3 ). The practical manifestation of constructive alignment principles for paramedic students is that every learning activity (eg. simulation experience) should have some form of formative feedback or assessment process (eg. simulation debrief) that aligns to the activity and its purpose and learning outcomes (eg. simulation learning outcomes). In turn the activities purpose and learning outcomes should be aligned to overall paramedic curriculum aims and objectives, which are in turn aligned to paramedic competencies or performance domains. In doing this, learning experiences such as simulations are specific, purposeful and focused and involve a formative feedback process. Learners are aided in construction and reconstruction of learning that is focused on the intended outcomes and paramedic competencies.

The PDM employs a construct of paramedic competencies adapted from the work of Tavares et al (42) that are intended to shape learning outcomes, the simulation experience and the focus of the debrief process. It is important to point out that the intention is not to utilise all eight of the paramedic competency dimensions in a single simulation experience, but rather to set one or two as the focus for the simulation experience and subsequent formative debrief. It is important to also point out that optimal simulation experiences and debrief should be learner centred and focussed on the needs of individual learners. While constructively aligned competencies or performance domains have a role in shaping learning outcomes in specific simulation experiences it is important to also allow for individual learning needs of learners to shape learning outcomes. Adopting a learner centred approach to the simulation experience and debrief will allow the blending of these two dimensions that shape the purpose and learning outcomes of the simulation experience (19).

The PDM was developed to address the need for a debrief model that would foster optimal learning from simulation experiences in the context of paramedic practice. It provides a practical aide memoire and has potential to provide a standardised evidenced based structure for debriefing paramedic simulations experiences. The model has a sound theoretical base drawn from current constructivism and experiential learning theory, reflective learning practices and constructive alignment curriculum theory. The next phase of research will be to trial the model. In particular pilot testing of the model may lead to further iterative revisions of the model based on paramedic educator and student end-user feedback. 


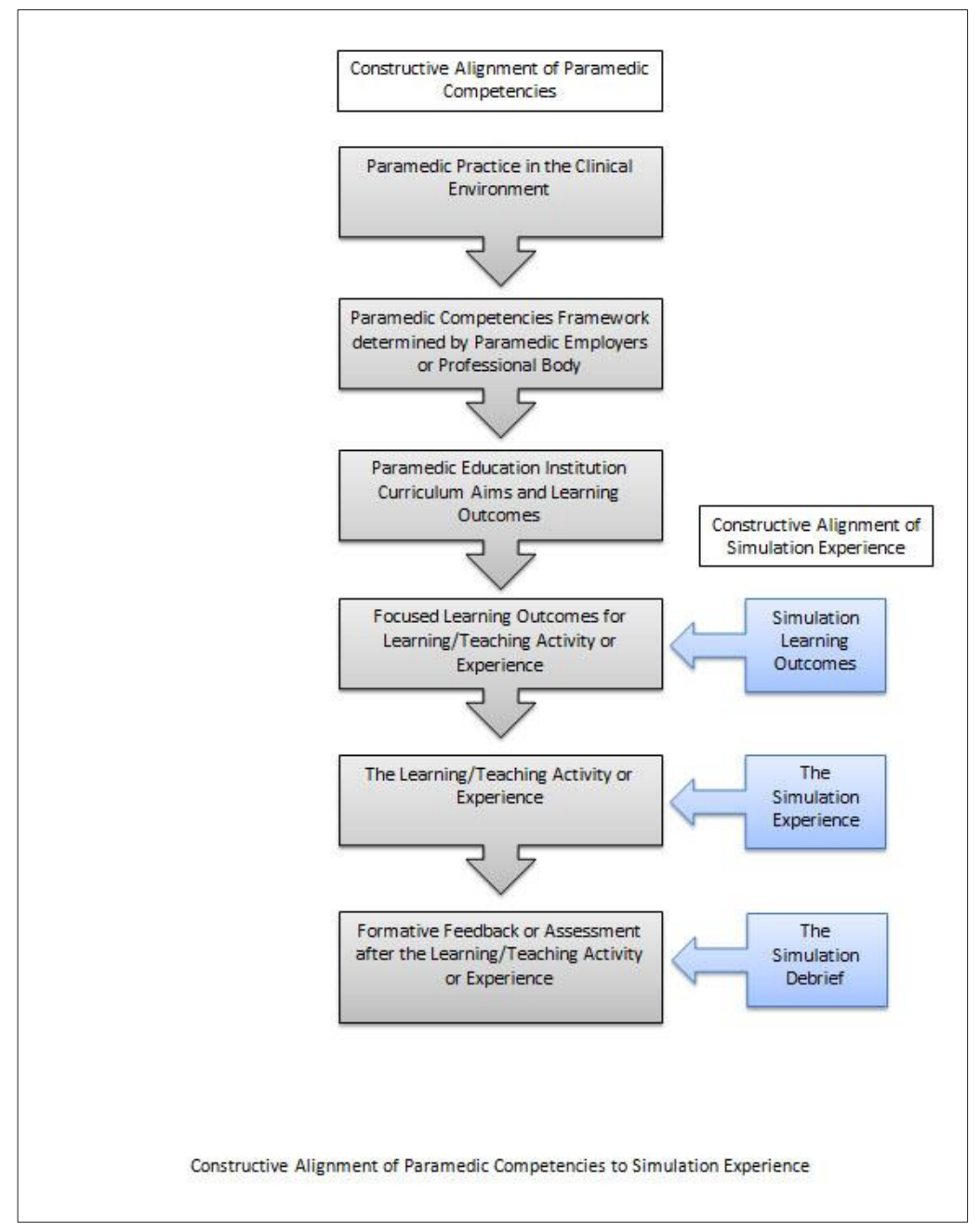

Figure 3. Constructive alignment of paramedic competencies to simulation

\section{Conclusion}

The PDM is the first simulation debrief model to propose and explore the use of a simulation debrief strategy that is unique to the field of paramedic simulation education and practice. The models framework has drawn from the research on best practice for conducting debriefs from other allied healthcare disciplines. Moreover, it proposes a framework for debrief that adheres to constructive alignment principles that ensures that a simulation learning experience and debrief is aligned to paramedic orientated learning outcomes that meets both learner needs as well as curriculum requirements. The PDM provides a framework that can ensure all key elements of an effective debrief are successfully achieved. The development of this model lays the foundation for further research in this area that will focus on validating its effectiveness and utility as a tool for guiding debrief facilitation. The PDM has potential to be utilised as a standardised strategy for facilitating simulation debriefs in paramedic education. As such, it contributes to the emerging body of knowledge supporting the use of simulation education in paramedic education and more specifically, the development of best practices with respect to simulation debriefing.

\section{Conflict of interest}

The authors report no conflicts of interest. Each author of this paper has completed the ICMJE conflict of interest statement.

\section{References}

1. Issenberg SB, McGaghie WC, Petrusa ER, Lee Gordon $D$, Scalese RJ. BEME: features and uses of high-fidelity medical simulations that lead to effective learning. A BEME systematic review. Med Teach 2005;27:10-28.

2. McKenna KD, Carhart E, Bercher D, Spain A, Todaro J, Freel J. Simulation use in paramedic education research (SUPER): a descriptive study. Prehospital Emerg Care 2015;19:432-40.

3. Ziv A, Wolpe PR, Small SD, Glick S. Simulation-based medical education: an ethical imperative. Acad Med 2003;78:783-8.

4. Garden A, Robinson B, Weller J, Wilson L, Crone D. Education to address medical error - a role for high fidelity patient simulation. N Z Med J 2002;115:133-4.

5. Murphy JG, Cremonini F, Kane GC, Dunn W. Is simulation based medicine training the future of clinical medicine? Eur Rev Med Pharmacol Sci 2007;11:1-8. 


\section{References (continued)}

6. Kolb D. Experiential learning: experience as the source of learning and development. Englewood Cliffs: Prentice Hall, Inc.; 1984.

7. Schon $\mathrm{D}$. The reflective practitioner: how professionals think in action. Aldershot: Ashgate Publishing Ltd., 1983.

8. Gibbs $\mathrm{G}$. Learning by doing: a guide to teaching and learning methods. London: Fell, 1988.

9. McKenna KD, Carhart E, Bercher D, et al. Simulation Use in Paramedic Education Research (SUPER): a descriptive study. Prehosp Emerg Care 2015;3127:1-9.

10. Abelsson A, Rystedt I, Suserud B-O, Lindwall L. Mapping the use of simulation in prehospital care: a literature review. Scand J Trauma Resusc Emerg Med 2014;22:22.

11. Power D, Henn P, Power T, McAdoo J, Hynes H, Cusack $S$. An evaluation of high fidelity simulation training for paramedics in Ireland. Int Paramed Pract 2013;2:11-8.

12. Williams B, Abel C, Ross L, Levett-Jones T. Simulation experiences of paramedic students: a cross-cultural examination. Adv Med Educ Pract 2016;7:181-6.

13. Neill MA, Wotton K. High-fidelity simulation debriefing in nursing education: a literature review. Clin Simul Nurs 2011;7:e161-8.

14. Mariani B, Cantrell MA, Meakim C, Prieto P, Dreifuerst KT. Structured debriefing and students' clinical judgment abilities in simulation. Clin Simul Nurs 2013;9:e147-55.

15. Dreifuerst $K$. Using debriefing for meaningful learning to foster development of clinical reasoning in simulation. $J$ Nurs Educ 2012:51:326-33.

16. Wickers MP. Establishing the climate for a successful debriefing. Clin Simul Nurs 2010;6:e83-6.

17. Biggs $J$, Tang $C$. Teaching for quality learning at university. 4th edn. Berkshire: McCraw Hill; 2011.

18. Rudolph JW, Simon R, Raemer DB, Eppich WJ. Debriefing as formative assessment: closing performance gaps in medical education. Acad Emerg Med 2008;15:1010-6.

19. Cheng A, Morse KJ, Rudolph J, Arab AA, Runnacles J, Eppich W. Learner-centered debriefing for health care simulation education: lessons for faculty development. Simul Healthc 2016;11:32-40.

20. Zigmont JJ, Kappus LJ, Sudikoff SN. Theoretical foundations of learning through simulation. Semin Perinatol 2011;35:47-51.

21. Arora S, Runnacles J, Ahmed M, et al. The London handbook for debriefing. London: Imperial College; 2011.

22. Jaye $P$, Thomas $L$, Reedy $G$. 'The diamond': a structure for simulation debrief. Clin Teach 2015;12:171-5.

23. Kolbe M, Weiss M, Grote G, et al. TeamGAINS: a tool for structured debriefings for simulation-based team trainings. Br Med J Qual Saf 2013;22:541-53.

24. Zigmont JJ, Kappus LJ, Sudikoff SN. The 3D model of debriefing: defusing, discovering, and deepening. Semin Perinatol 2011;35:52-8.
25. Eppich W, Cheng A. Promoting Excellence and Reflective Learning in Simulation (PEARLS): development and rationale for a blended approach to health care simulation debriefing. Simul Healthc 2015;10:106-15.

26. Bajaj K, Meguerdichian M, Thoma B, et al. The PEARLS Healthcare Debriefing Tool. Acad Med 2018;93:336.

27. Cheng A, Grant V, Robinson T, et al. The Promoting Excellence and Reflective Learning in Simulation (PEARLS) approach to health care debriefing: a faculty development guide. Clin Simul Nurs 2016;12:419-28.

28. Fanning RM, Gaba DM. The role of debriefing in simulationbased learning. Simul Healthc J Soc Simul Healthc 2007;2:115-25.

29. Steinwachs B. How to facilitate a debriefing. Simul Gaming 1992:23:186-95.

30. Arora S, Ahmed M, Paige J, et al. Objective structured assessment of debriefing: Bringing science to the art of debriefing in surgery. Ann Surg 2012;256:982-8.

31. Pendelton D, Schofield T, Tate P, Havelock P. The consultation: an approach to learning and teaching. Oxford: Oxford University Press, 2003.

32. Rudolph JW, Simon R, Dufresne RL, Raemer DB. There's no such thing as "nonjudgmental" debriefing: a theory and method for debriefing with good judgment. Simul Healthc 2006;1:49-55.

33. Smith-Jentsch KA, Cannon-Bowers JA, Tannenbaum SI, Salas E. Guided team self-correction: impacts on team mental models, processes, and effectiveness. Small Gr Res 2008:39:303-27.

34. Jones I, Alinier G. Introduction of a new reflective framework to enhance students ' simulation learning : a preliminary evaluation. Blended Learn Pract 2009;6:1-19.

35. Starfield $B$. Is patient-centered care the same as personfocused care? Perm J 2011;15:63-9.

36. Stone J. What is person-centred care and why is it important? Behav Res Ther 2006;308-13.

37. Paramedics Australasia. Australasian Competency Standards for Paramedics. 2011;1-11. Available at: www.paramedics.org/content/2011/10/PA_AustralasianCompetency-Standards-for-paramedics_July-20111.pdf

38. Health Professions Council. Standards of proficiency: paramedics [Internet]. 2014. Available at: www.hpc-uk.org/ assets/documents/1000051CStandards_of_Proficiency_ paramedics.pdf

39. Paramedic Association of Canada. National occupational competency profile for paramedics. 2011;172. Available at: www.paramedic.ca/uploaded/web/documents/2011-10-31Approved-NOCP-English-Master.pdf

40. Standards New Zealand. New Zealand standards for ambulance and paramedical services 8156. 2008. Available at: https://shop.standards.govt.nz/catalog/8156:2008(NZS)/ scope

41. The Council of Ambulance Authorities. Paramedic Professional Competency Standards, v2. Flinders Park, 2010. 


\section{References (continued)}

42. Tavares W, Boet S, Theriault R, Mallette T, Eva KW. Global rating scale for the assessment of paramedic clinical competence. Prehospital Emerg Care 2013;17:57-67.

43. Tavares W, LeBlanc VR, Mausz J, Sun V, Eva KW. Simulation-based assessment of paramedics and performance in real clinical contexts. Prehospital Emerg Care 2014;18:116-22.
44. Townsend R, Luck M. Applied paramedic law and ethics Australia and New Zealand. Chatswood: Elsevier, 2013.

45. Doyal L, Gillon R. Medical ethics and law as a core subject in medical education. BMJ 1998;316:1623.

46. Williams B, Dousek $S$. The satisfaction with simulation experience scale (SSES): a validation study. J Nurs Educ Pract 2012;2:74-80.

47. Cantrell MA. The Importance of debriefing in clinical simulations. Clin Simul Nurs 2008;4. 